\title{
COMPARACIÓN DE VARIAS TÉCNICAS COPROPARASITOLÓGICAS PARA EL DIAGNÓS- TICO DE GEOHELMINTIASIS INTESTINALES
}

\author{
Fidel Angel NUÑEZ-FERNÁNDEZ (1), Esperanza SANJURJO GONZALEZ (2) \& Carlos M. FINLAY VILLALVILLA (3).
}

\section{RESUMEN}

Se procesan "a ciegas" 511 muestras de heces por las técnicas de Kato-Kat., Willis, Ritchic y directo. Al comparar los resultados obtenidos en cl diagnóstico de Trichuris trichiura, Necator americanus y Ascaris lumbricoides, vimos que la de Kato-Katz fué más sensible, revelando el mayor número de casos, siguiéndole en orden sucesivo, el Willis, el Ritchie, y el examen directo. Si se tiene en cuenta que cl método de Kato-Katz es además cuantitativo, podemos recomendar-lo como técnica de elección para el diagnóstico de las geohelmintiasis intestinales.

UNITERMOS: Técnicas coproparasitológicas, Kato-Kak, Ritchic, Willis, Dirccto.

\section{IN'TRODUCCION}

El diagnóstico de la mayoria de las infecciones por parásitos intestinales se fundamenta sobre todo en el examen microscópico de la materia fecal y ha experimentado pocos cambios en los últimos 50 años $^{18,19}$. Sin cmbargo se pudieran cilar algunas $\mathrm{ex}-$ cepciones como son la técnica de Harada-Mori para coprocultivos de larvas? y el frotis grueso en cclofán de Kato.

La tčenica de Kato, fuc introducida en 1954 por KATO \& MIURA ${ }^{8}$ y fué ampliamente cmpleada en programas de control, que se realizaron en la década del $50 \mathrm{cn}$ Japón. Sin cmbargo no se difundió su conocimiento en el mundo, hasta el año 1966, donde aparece una publicación en inglés de KOMIYA \& KOBAYASHI con una evaluación de las ventajas y limitaciones del método ${ }^{10}$. En 1968, aparece una publicación de MARTIN \& BEAVER, donde sc realiza una cvaluación de la técnica y se le introducen 3 importantes modificaciones, que consistian en remover las fibras de la muestra fecal mediante un fino tamiz, la discminación unilonne del frotis, y la prevención del sobreaclaramiento de la preparación al determinar el ticmpo óptimo para la lectura". En 1972, KATZ introduce una nueva modificación al método, convirtiendo la lécnica de gravimétrica a volumétrica y haciendo posible su cmpleo para trabajos de campo". En esta forma se difunde cl método, por la OMS para el diagnóstico cuali-cuantiativo de las infeceiones intestinales humanas por geohelmintos ${ }^{17}$. En cl presente estudio nos propusimos cvaluar la efectividad del método en nucstras condiciones de trabijo al compararlo con las técnicas que más frecuentemente empleamos.

\section{MATERIALES Y METODOS}

Se procesaron 511 muestras de heces, procedentes de 511 personas de una comunidad rural, por varias técnicas coproparasitológicas: directo', WILLIS $^{20}$ modificado por BASNUEVO ${ }^{6}$ y RITCHIE ${ }^{14}$. El método de Kato-Kat\% se desarolló según descripción de la OMS ${ }^{17}$, se empleó cl kit de Ovo-Fec de la Bochringer Mannheim Bioquímica S.A. de Brasil, y en el caso de hallar huevos de ancylostomídeos por cualguier técnica se realizó la siembra por cl método de Harada-Mori y la posterior identilicación de las larvas mediante las claves cstablecidas por LITTLE'. Los procederes fucron realizados por 2 lécnicos calificados, que trabajaron "a ciegas" las 4 técnicas diagnósticas para comparar despućs sus resultados.

(1) Especialista en Microbiologia (Parasitologia). Instituto de Medicina Tropical "Pedro Kouri".

(2) Especialista de II grado en Epidemiologia. Instituto de Medicina "lropical "Pedro Kouri".

(3) Candidato a Dr. en Ciencias. Subdirector de Parasitologia. Insituto de Medicina Tropical "l'edro Kouri".

Correspondencia para: Dr. Fidel Núnez lemández. Instituto de Medicina Tropical "Pedro Kouri". Ave 15 y 200, Aptdo. Postal 601, Mlaya, C. Ilabana, Cuba. 'Telex: CU-IPK-5119)2 512341 
NÚNEZZ-FERNÁNDEZ, F. A.; SANJURJO GONZALIZZ, E. \& FINLAY VILLLALVILLA, C. M. - Comparación de varias técnicas coproparasitológicas para el diagnóstico de geohelmintiasis intestinales. Rev. Inst. Med. trop. S. Paulo, 33 (5): 403-406, 1991.

El análisis estadístico se realizó en una microcomputadora IBM AT, utilizando cl paquete de programas Microstat, y se empleó como estadigrafo de comparación, el test de proporciones, teniendo siempre en cuenta, la coincidencia entre categorias.

\section{RESULTADOS}

En la Tabla 1, al comparar cl método de KatoKatz, con el de Willis, cncontramos que el primero, obtuvo una mayor positividad, para cl diagnóstico de Trichuris trichiura y Ascaris lumbricoides $(p<0,01)$, sin cmbargo, no se cncontraron diferencias significativas en cuanto a la positividad de ambos métodos para el hallazgo de Necator americanus ( $>00,05)$. Al comparar, el Kato con el Ritchic, encontramos que el primer método, resultó significativamente más sensible para el diagnóstico de $T$. trichiura, $N$. americanus $(p<0,01)$ y $\mathbf{A}$. Iumbricoides $(\mathrm{p}<0,05)$. Cuando se comparó la positividad por especies entre el método de Kato-Katz y cl examen directo, se encontró que la frecuencia de casos hallados por el primer método, fue significativamente mayor en todos los helmintos estudiados, $\mathrm{T}$. trichiura, N. americanus, y A. lumbricoides $(\mathrm{p}<0,01)$.

Cuando se analizó la coincidencia de las diferentes técnicas coproparasitológicas con el método de Kato-Katz observamos que se comportó según la sensibilidad de las mismas. La técnica de Willis, fué la más sensible, de las comparadas con el Kato, por lo que presentó la mayor coincidencia, siguiéndole en orden sucesivo el Ritchic y el directo. Sin embargo al analizar el comportamiento de la media de los conteos de huevos en los casos coincidentes, vimos que se comportó en forma inversamente proporcional. es decir, ha medida que la técnica es menos sensible, la media de los conteos de huevos será mayor, detectándose solamente las infecciones más intensas con mayores cantidades de huevos.

\section{DISCUSION}

La técnica de Kato-Kav, resultó más sensible que la de Ritchie y el directo para cl diagnóstico de las geohelmintiasis. Sin embargo, la técnica de Willis solo fué superada evidentemente por la de Kato-Kat\%, en el diagnóstico de $\mathrm{T}$. trichiura ya que en el caso de N. americanus, ambas técnicas demostraron similar sensibilidad. Consideramos que para el diagnóstico de A. lumbricoides, el Willis y el Kato, no pudieron ser comparables, al ser todos los huevos hallados infecundos, con un mayor peso específico que los fértiles y $\mathrm{cl}$ Willis basa su capacidad diagnóstica, en el menor peso específico de los huevos contenidos en una solución salurada de densidad 1200, lo que permite concentrar por flotación solo a los huevos más ligeros que el medio.

El método del formol-eter de Ritchic se señala como un método eficiente para recuperar quistes y huevos de helmintos ${ }^{1,}$, y el de flotación en salmuera de Willis, aunque es particularmente recomendado para recuperar huevos de ancylostomideos, se considera como un proceder útil, para $A$. lumbricoides y $T$, trichiura, pues practicamente todos los huevos de nemátodos se concentran con esta técnica ${ }^{1,2,12,16}$. La técnica de Kato ha sido comparada con otras técnicas, sin embargo en nuestro conocimiento no existen trabajos previos, donde se compare adecuadamente con los mélodos de Ritchic y Willis, que son los más cm-

TABLA 1

Comparación entre las técnicas de Kato, Willis, Ritchic y directo para diagnóstico de gcohelmintos

\begin{tabular}{lrcccc}
\hline & \multicolumn{4}{c}{-Positivos por cada técnica- } & Willis \\
Parasito & Total* & Kato-Kat\% & Ritchic & Dirccto \\
& 139 & $133(95,7)$ & $67(48,2)$ & $37(26,6)$ & $\mathrm{N}^{\circ}(\%)$ \\
\hline Trichuris trichiura & 57 & $44(77,2)$ & $42(73,7)$ & $17(29,8)$ & $7(12,3)$ \\
Necator americanus & 7 & $7(100)$ & $2(28,6)$ & $4(57,1)$ & $4(57.1)$ \\
Ascaris lumbricoides & & & &
\end{tabular}

* Positivos por cualquier técnica 
NÚNEZZ-FERNÁNDEZ, F. A.; SANJURJO GONZALEZ, E. \& FINLAY VILLALLVILLA, C. M. - Comparación de varias técnicas coproparasitológicas para el diagnóstico de geohelmintiasis intestinales. Rev. Inst. Med. trop. S. Paulo, 33 (5): 403-406, 1991.

pleados cn nuestro medio. MARTIN Y BEAVER, encontraron la técnica de Kato, tan sensible como el método de filtración de Bell, cl de flotación en sulfato de zinc y el de sedimentación de Hoffman ${ }^{11}$, mientras que GARCIA y colaboradores hallaron el Kato más sensible que cl directo y $\mathrm{cl}$ Teleman-Rivas, para el diagnóstico de las gcohelmintiasis ${ }^{5}$. PAGLIUSI y colaboradores, compararon el Kato-Katz con otros métodos de conteos de huevos, como el de Mc Master y Stoll-Haushecr y concluyeron que el primero fuc el más cfectivo, no solo por la mayor sensibilidad sino también por la capacidad de revelar las tasas más elevadas ${ }^{13}$.

El método de Kato-Kay no solo nos brinda el diagnóstico cualitativo, sino que también nos permite conocer la intensidad de la infección a través del contco de los huevos excretados, y clasificar la infección en ligera, moderada o intensa. Además este resulta el método idónco por su sensibilidad, para cl diagnóstico de T. trichiura, helminto más frecuente en $\mathrm{Cuba}^{4,15}$ y en el área del Caribe ${ }^{3}$. Si a todo lo anteriormente expresado le agregamos que las técnicas de diagnóstico coproparasitológịco son las de clección en programas de control del parasitismo intestinal, por su bajo costo, simplicidad y sensibilidad, condiciones que cumple cl Kato-Katz, nos permitc recomendar este método como el de clección para cl diagnóstico de las geohclmintiasis intestinales.

\section{SUMMARY}

Comparison of several coproparasitological techniques for diagnosis of soil-transmitted helminthiasis

Five hundred and eleven sumples of feces were examined by several coproparasitological techniques: Kato-Katz, Willis, Ritchic and direct wet mount. It was found that Kato-Kat. was the most reliable technique for the diagnosis of Trichuris trichiura, Necator americanus, and Ascaris lumbricoides, demonstrating the largest number of cases. Willis, Ritchic and direct wet mount were effective in that order. Taking into account that KatoKatz is a quantitative method, it should be recommended in the diagnosis of soil-transmitted helminthiasis.

\section{AGRADECIMIENTOS}

Al professor Naftale Kat\%, de Brazil por sus sabios consejos.

\section{REIIERIENCIAS BHILIOGRAIICAS}

1. ASII, L. R. \& ORIHEL, T. C. - Parasites: a guide to laboratory procedures and identification. Chicago, ASCP Press, 1987.

2. BALOWS, A. \& IIAUSLIER, W. J., ed. - Bacterial, mycotic and parasitic infections. 6th. cd. Washington, American Public Health Association, 1981.

3. BUNDY, D. A. P. - lipidemiological aspects of Trichuris and trichuriasis in Caribbean communities. Trans. roy. Soc. trop. Med. Hyg. ,80: 706-718, 1986.

4. IERRIER, H.; BLRROA, M.: TIEJEIRO, A. \& SOTOLOAGO, I: - Fincuesla Nacional de Morbilidad por Parasitismo Intestinal en Cuba. Rev. Cub. Hig. Epidem., 13: $118-119,1975$

5. GARCIA, J. A.: MAR'TIN, A. M. \& PIRIEZ, M. J. - Valoración de los métodos utilizados en el diagnóstico de parasitosis intestinales. Laboratorio, 79: 473, 1985.

6. GRUPO NACIONAL DH LABORATÓRIOS CLINICOS. MINSAP - Manual de técnicas para laboratórios clínicos. La Ilabana, Ciencia y T'écnica, 1969. p. 151-153.

7. IIARADA, Y.\& MORI, O. - A new method for culturing hookwonn. Yonago Acta med., 1: 177-179, 1955.

8. KA'O, K. \& MIURA, M. - Comparative examinations. (Japanese texl). Jap. J. Parasit., 3: 35, 1954.

9. KAT\%, N.; CHAVE\%, A. \& PBLLLGRINO, J. - A simple device for quantitative stool thick-smear techinique in schistosomiasis mansoni. Rev. Inst. Med. trop. S. Paulo, 14: $397 \cdot 400,1972$.

10. KOMIYA, Y.\& KOIBAYASIII, A. - livaluation of Kato's thick smear technique with a cellophane cover of helminth eggs in fueces. Jap. J. med. Sci. IBiol., 19: 59-64, 1966.

11. MARTIN, L. K. \& BI:A VIBR, P. C. - Evaluation of Kato's thicksmear lechnique for quantitative diagnosis of helminth infections. Amer. J. trop. Med. Hyg., 17:382391,1968 .

12. MELVIN, D. M. \& BROOKI:, M. M. - Laboratory procedures for the diagnosis of intestinal parasites. Allanta, Burcau of Laloratorics. Iaboratory Training \& Consultation. Div., U. S. Dept. of IIealth, Education \& Welfare, Public llealth Service, 1981.

13. PAGIJUSI, V. I.; TURRI, L. S.; CAMPOS, R.; AMATO NEIO, V.; BAILI.O', $\Lambda$. A.; SILVA, P. L. \& GOMI:Z, A. li. C. - Contagem de ovos de helmimos nas fezes, pelo método de Mc Master, Stollllausheer e Kato-Katz. In: JORNADA CIEN"IÍFICA DO IASITTUTO DE MEDICINA 'IROPICAL dDI: SÃO PAULO, 3., São Paulo, 1989. (Rev. Inst. Med. trop. S. Paulo, 31 (supl. 7): S61, 1989)

14. RITCIIII:, L. S. - An ether sedimentation for routine stool examinations. IBull U.S. Army med. Dep., 8: 326, 1948.

15. SANJURJO, I.; RODRIGUI\%, M.; BRAVO, J. R.; 
NÚÑEZ-FERNÁNDEZ, F. A.; SANJURJO GONZALFZ, F. \& FINLAY VILLAL.VILLA, C. M. - Comparación de varias técnicas coproparasitológicas para el diagnóstico de geohelmintiasis intestinales. Rev. Inst. Med. trop. S. Paulo, 33 (5): 403-406, 1991.

FINLAY, C. M.; SILVA, L. C.; GÁLVEZ, M. D. \& CORDOVI, R. - Encuesta nacional de parasitismo in. testinal. Habana, Informe Técnico Inédito, Instituto de Medicina Tropical "Pedro Kouri". 1984.

16. TIIIENPONT, D.; ROCHEITIE, F. \& VANPARIJS. O. F. J. - Diagnóstico de las helmintiasis por medio del examen coprológico. Belgica, Janssen Rescarch loundation, 1979.

17. W.II.O.: Cellophane Faccal thicksmear examination technique (Kato) for diagnosis of intestinal schistosomiasis and gastrointestinal helminth infections. PDP/83.3, 1983.
18. W. H. O. - Expert Committee: prevention and control of intestinal parasitic infections. Wld. Hith. Org. techn. Rep. Ser., (749), 1987.

19. W. H. O. - Scientific Working Group: Intestinal protozoan and helminthic infections. Wld. Hith. Org. techn. Rep. Ser., (666), 1981.

20. WILI.IS, H. II. - A simple levitation method for the delection of hookworm ova. Med. J. Aust., 8: 375-376, 1921.

Reccbido para publicação em 28/01/1991. Accito para publicação em 23/05/1991. 